\title{
Causal Factors in Implementing Environmental Regulation: Evidence from the Erosion and Sediment Control Plan in Malaysia
}

\author{
A.S.Asnor ${ }^{1}$, R.A.Rahman ${ }^{\text {* }}$ and S.W.Ahmad ${ }^{2}$ \\ ${ }^{1}$ Faculty of Civil Engineering Technology, Universiti Malaysia Pahang, 26300 \\ Gambang, Pahang, Malaysia \\ ${ }^{2}$ Department of Civil Engineering, College of Engineering, Universiti Malaysia Pahang, 26300, Kuantan, \\ Pahang, Malaysia \\ *Email: arahimirahman@ump.edu.my
}

\begin{abstract}
Erosion, and sedimentation can have a significant impact on the environment. Therefore, the Erosion and Sediment Control Plan (ESCP) is necessary to reduce those processes' adverse effects. Even though implementing ESCP is compulsory, we still have implementation issues in practice. This study aims to investigate the causal factors that are affecting ESCP implementation in Malaysia. To achieve that objective, data are collected through individual interviews with industry practitioners related to ESCP implementation. Then, the data are analyzed using thematic analysis. The challenges for implementing ESCP can be divided into two (2) internal and external challenges. Internal challenges consist of lack of competency, lack of knowledge, communication, attitude, awareness, planning, design, installation, and maintenance. In contrast, external challenges include management, financial, enforcement, and communication medium. This research contributes to the body of knowledge by providing insights into ESCP implementation, which could help develop policies and strategies to tackle the challenges. The findings of this research will help both researchers and practitioners to develop strategies that contribute to the sustainability of earth resources by reducing detachment of a portion of soil profiles from soil surfaces into soil particles (i.e., erosion); and transportation and deposition of soil particles (i.e., sedimentation) in construction projects.
\end{abstract}

Indexed Terms- Sustainable development, sustainable construction, decision making, developing countries, environmental regulation.

\section{INTRODUCTION}

Erosion and sedimentation are natural processes that occur every day throughout the country due to wind and water. However, the effect of natural erosion is usually only noticeable on a geologic time scale. Soil or surface disturbance, including construction, agriculture, or logging, can significantly increase the amount of sediment loss from the site because of erosion [1]. This change can increase the risk of flooding, alter hydrology, and destroy the hydric vegetation. Sediments can also smother fish or amphibians nesting sites or cover mussel beds that filter substantial amounts of water pollutants that eventually become our drinking water [1]. Therefore, sufficient on-site sediment and erosion control in the construction industry provides many environmental, social, and economic benefits, including improved occupational health and safety on the building site, clean-up costs, wet weather working conditions, building completion, and downtime, and waterways.

The erosion and sediment control plan (ESCP) is a plan that details temporary measures to be implemented during the construction phase. It may include permanent means to remain in place once the erosion and sedimentation development control measures are completed. Specifically, ESCP adopts the best management practices for preventing the loss of sediment from a building site (or any other site) during most storm events by leaving original vegetation whenever possible, restoring vegetative 
cover as soon as possible, and controlling clean water diversions, silt fences, and sediment basins. The practical ESCP objective is to prevent controllable erosion and minimize the adverse effects of on-site sediment transport. In Malaysia, performing the ESCP is necessary for all construction projects by adhering to the "Guideline for Erosion and Sediment Control in Malaysia" provided by the Department of Irrigation and Drainage (DID). However, although implementing ESCP is compulsory, the Malaysian construction industry has issues in practice related to implementing ESCP.

This study's objective is to investigate the factors that influence the success of implementing ESCP. To achieve that objective, data on the challenges and success factors for implementing ESCP are collected through open-ended individual interviews with industry practitioners from the construction industry. The collected data are then analyzed using thematic analysis to identify themes that can represent the whole data. This study contributes to the existing sustainable construction management body of knowledge by providing the causal factors for ESCP implementation. These factors can help industry practitioners develop strategies to ensure the success of ESCP implementation in Malaysia. Successful ESCP implementation is essential to protect the environment by building up Malaysia into a developed county.

\section{LITERATURE REVIEW}

\subsection{Challenges in implementing environmental regulation}

Researchers and practitioners investigate environmental regulation topics from construction project management, environmental, and law. There are numerous challenges or problems in implementing environmental law, including land disturbance in erosion and sediment control on construction sites, costly and expensive to control erosion, specifications that require deep understandings of erosion and sedimentation processes and their effectiveness, and incorrect installation and maintenance from the Department of Environment (DOE) of Malaysia. Also, the results of analyzing the economics of sediment control suggest that certain constraints limit project team members from complying with regulatory installation and maintenance standards [2]. Another study indicates several types of difficulties in adhering to environmental regulation, including economic and institutional challenges, after examining the characteristics, limitations, costs, and effectiveness of various sediment management strategies [3]. These difficulties can arise when issues emerge from design flaws and missing information [4]. Also, the complications can be associated with technical challenges consisting of limited knowledge and resources (e.g., shortage or lack of skilled staff and equipment to perform data collection, sediment control work planning, and design) appear to be one of the main barriers to environmental performance requirements in construction [5]. Conversely, challenges due to social and political complications are also affecting the environmental sustainability of construction projects [6]. In other words, the level of stringency and the intensity of the control activities are a powerful stimulus to improve the environmental and competitive performance in our construction industries [7]. Therefore, several studies suggest approaches to enhance available plans, including providing practical implications and frameworks for implementing environmental regulatory procedures and future policy formulation in developing countries. In conclusion, strategic planning for executing environmental laws is necessary to enhance the success and reduce its implementation challenges.

\subsection{Erosion and Sediment Control Plan (ESCP)}

Erosion and sedimentation are severe environmental problems that we are facing. Erosion and sedimentation are natural processes that occur every day throughout the country due to wind and water. In Malaysia, we have unique tropical weather all year round. The climate is often quite humid due to its proximity to water, increasing local temperature, and the highlands nevertheless experience cooler temperatures. The unique tropical climate came with two seasons, which is monsoon season and dry season. In the dry season, wind erosion can become a problem when the soil is loose, dry, bare, or almost bare, and the wind speed exceeds the threshold speed for soil particle movement [8]. However, 
during the monsoon season, the high rainfall amounts. Therefore, it is necessary to exercise strong control over rivers' discharge and, consequently, the transport of sediments, resulting in major flooding and deposition of sediments caused by erosion. Humans get over $99.7 \%$ of their food (calories) from the land and less than $0.3 \%$ from the oceans and other aquatic ecosystems. Human-made erosion is one of the main factors in environmental problems, mostly in erosion and sediment. Another study discussed the contrasts between man-affected catchments and those with significant cultivation areas in the humid tropics in the Cameron Highlands of West Malaysia, stating that a catchment with $94 \%$ of its natural vegetation area had a sediment yield of $21.1 \mathrm{~m}^{3} / \mathrm{km}^{2} / \mathrm{yr}$. A nearby catchment with only $64 \%$ of the natural forest area had a yield of $103.1 \mathrm{~m}^{3} / \mathrm{km}^{2} / \mathrm{yr}$. Ground surface disturbance, including construction, agriculture, or logging, significantly increases sediment loss from the site due to erosion. In Peninsular Malaysia, steep mountainous land has been developed for agriculture vegetables, tea, floriculture, and fruit [9]. This will increase the erosion and sediment problem because of decreases in terrain, preventing the rainfall from directly hitting the soil. Approximately 10 million hectares of cropland are lost each year worldwide due to soil erosion, reducing the available farmland for food production [9]. Moreover, sediments can also smother fish or amphibians nesting sites or cover mussel beds that filter substantial amounts of water pollutants that eventually become our drinking water [1]. In erosion and sediment control, the study finds that erosion and sediment can be categorized into two categories, which are natural erosion and human-made erosion. Natural erosion is occurred because of weather and the changing of the season in Malaysia, but the common factor of erosion problems coming from humanmade erosion. Human-made erosion comes from ground surface disturbance such as construction, agriculture, or logging. This erosion problem can cause severe environmental issues such as water pollutants that eventually become our drinking water. Therefore, ESCP is vital in ensuring the sustainability of Malaysia's ecosystem.

\subsection{ESCP in Malaysia}

In Malaysia, researchers have explored various topics related to implementing ESCP, including its challenges and success factors. One study suggests that the challenges for implementing ESCP in Malaysia's construction industry includes managing and constructing ESCP devices. Another research indicates that when setting up ESCP devices' requirements, the tools are usually prescribed based on readily available technical solutions rather than assessing environmental performance [5]. A study has also identified the challenges and barriers of ESCP implementation through case studies, including the urbanization and infrastructural development of the soil erosion associated with a building project at Bukit Kiara in Kuala Lumpur. [15]. While these studies provide insights into the factors of ESCP, the results also illustrate that different factors are affecting the local construction sector. Also, several of those studies may not capture additional factors that might affect the industry or acquire in-depth insights from the industry practitioners. Questionnaire surveys are standardized and inflexible to changes throughout the data collection.

\subsection{Positioning of this study}

There is limited understanding in the literature about the contributing factor for the successful implementation of ESCP in Malaysia. Therefore, this study fills this gap by identifying factors and success factors affecting Malaysia's ESCP through individual interviews with industry practitioners and policymakers. Although interviews have difficulties in generalization, this alternative approach provides opportunities to acquire unique perspectives, practical knowledge, and experiences from research participants on problems and success factors related to implementing ESCP. 


\section{METHODOLOGY}

In this research, the data collection involves the individual interview method, while the data analysis consists of using the thematic analysis to analyze the collected data.

\subsection{Data collection}

The study uses the individual interview method because there is inadequate information on the challenges and success factors for implementing ESCP. Numerous studies use individual interviews to study environmental behaviors, such as studying the consumption patterns and environmental awareness in formal and informal communities in Suva, Fiji Islands [11], and decision analysis interviews in environmental impact assessment [12]. Using this qualitative method, researchers manage to obtain the necessary data to answer their research objectives.

The target population for the interviews is professionals that are involved in ESCP implementation, including officers from the Department of Environment, the Department of Irrigation and Drainage, and Infrastructure Department of City Halls, City Councils, Municipal Councils, and District Councils, and engineers or environmental officers of contractors and consultant firms. Additionally, the respondents should be individuals that are experienced in implementing ESCP in construction projects. These criteria will give us appropriate data on finding the problems and solutions for implementing ESCP in the construction industry. Interviewee that meets these criteria will create useful data and reducing the outliers of the trends of the data. The interview involves twenty individuals that fit the predetermined criteria to mitigate bias and validity threats inherent in qualitative research [13]. Table 1 summarizes the respondents' sector, position, and organization.

Table 1: List of interviewees

\begin{tabular}{ccccc}
\hline ID & Sector & Stakeholder & Position & Organization \\
\hline 1 & Public & Law enforcer 1 & City Council Engineer & City Council \\
2 & Public & Law enforcer 2 & Environmental Officer & Public Work Department \\
3 & Public & Law enforcer 3 & Environmental Officer & Public Work Department \\
4 & Public & Law enforcer 4 & DID Officer & Department of Irrigation and Drainage \\
5 & Public & Law enforcer 5 & DID Officer & Department of Irrigation and Drainage \\
6 & Public & Law enforcer 6 & DID Officer & Department of Irrigation and Drainage \\
7 & Public & Law enforcer 7 & Engineer & Department of Irrigation and Drainage \\
8 & Private & Contractor 1 & Environmental Officer & Contractor \\
9 & Private & Contractor 2 & Environmental Officer & Contractor \\
10 & Private & Contractor 3 & Environmental Officer & Contractor \\
11 & Private & Contractor 4 & Environmental Officer & Developer \\
12 & Private & Contractor 5 & Environmental Officer & Contractor \\
13 & Private & Consultant 1 & Hydrology Engineer & Hydrology Consultant \\
14 & Public & Consultant 2 & Environmental Officer & Consultant under DOE \\
15 & Private & Consultant 3 & Environmental Officer & Consultant \\
16 & Private & Consultant 4 & Environmental Officer & Consultant \\
17 & Public & Engineer 1 & PWD Engineer & Public Work Department \\
18 & Private & Engineer 2 & Hydrology Engineer & Hydrology Consultant \\
19 & Private & Engineer 3 & Site Engineer & Developer \\
20 & Private & Engineer 4 & Engineer & \\
\hline & & & & Contractor \\
\hline
\end{tabular}


The interview sessions are approximately around 60 minutes. Before the interview started, the study's background and objectives are introduced. The topic's title and questions are printed on an A4 paper and handed to interviewees to refer to it while answering. This process ensures the respondents understand the topic and give answers according to the issue. However, any explanation that might be related to the potential answers is avoided to prevent bias among the respondents. The interviews also involved providing follow-up questions to acquire more details and information and avoid misunderstanding on the interviewees' responses. After the meeting, a summary of the discussion is shared with the interviewee for verification purposes. Here, respondents can check whether there is some misinformation or misinterpretation and provide feedback as necessary. Then, the summaries are revised and repeated until the respondents are satisfied.

\subsection{Data analysis}

The data from the interview responses are analyzed using thematic analysis. Thematic analysis is one of the most common forms of analysis in qualitative research. This method will emphasize pinpointing, examining, and recording the pattern or themes within the data. Therefore, this method allows researchers to find patterns within the data and create new themes representing the whole data. In this regard, thematic analysis is also adopted by Rahman and Ayer [14] and Radzi et al. [15] to analyze qualitative data associated with construction management topics.

\section{RESULTS AND DISCUSSION}

Table 2 and Table 3 show the challenges and success factors for implementing ESCP. The subsequent subsections discuss those challenges and success factors.

\subsection{Challenges for implementing ESCP}

The challenges for implementing ESCP can be divided into two (2) internal and external challenges. Internal challenges consist of lack of competency, lack of knowledge, communication, attitude, awareness, planning, design, installation, and maintenance. In contrast, external challenges include management, financial, enforcement, and communication medium.

\subsubsection{Internal challenges}

For internal challenges, the design has the highest number of hits compared to the other problems. This challenge involves project team members who do not follow the specifications and do not plan for ESCP implementation to save cost. In detail, consultants perceived that contractors are not implementing ESCP according to the provided design. On the other hand, contractors viewed consultants are designing the ESCP not according to the site condition but based on theory. This misunderstanding between both parties results in either inappropriate and dislocated ESCP devices or designs that differ from the site condition. In other words, this lack of collaboration between the designers and constructors can cause problems for a project [16]. This result resonates with a prior finding from another study that analyses the economics of sediment control at construction sites [2]. In other words, this finding suggests that the challenge of designing ESCP can be associated with miscommunications between contractors and consultants, and the existence of a gap between theory and reality that might not be addressed even with good communications.

While environmental regulation is crucial, respondents suggest that its implementation in practice is challenging if there is a lack of knowledge or competency among project team members. For example, this lack of knowledge can result in developers and owners that assign consultants without adequate experience and knowledge in environmental regulation due to personal preferences. Also, contractors often quote low prices during the bidding process from not knowing the work scope for implementing ESCP. Conversely, labor workers without the necessary competencies can result in unforeseen challenges during the construction of ESCP devices. Also, respondents suggest that while some 
engineers can design ESCP, there is still a lack of engineers with the necessary competencies to acquire the Certified Professional in Erosion and Sediment Control (CPESC).

Besides that, attitude and awareness are also part of the internal challenges for implementing environmental regulation in the construction industry. Specifically, challenges associated with attitude include assuming ESCP (i.e., environmental regulation) is not mandatory and perceiving ESCP as an activity that wastes resources. This type of attitude results in contractors not installing ESCP devices according to the standards and specifications. This results in the emergence of a competent person with the CPESC that signs ESCP designs without checking and analyzing the related documents. On the other hand, similarly to another study, a lack of awareness of environmental regulation is often associated with the local culture towards sustainable development [17]. This lack of awareness results in contractors and consultants that fail to implement environmental regulation from not knowing the appropriate actions. In other words, lack of awareness and lack of knowledge can fail in implementing environmental regulation. The findings also suggest that the construction industry focuses on activities that benefit construction projects directly rather than events that contribute to sustainability. Therefore, it is vital to educate all individuals on sustainability to ensure the right attitude and awareness in their future works.

Table 2: Challenges in implementing ESCP

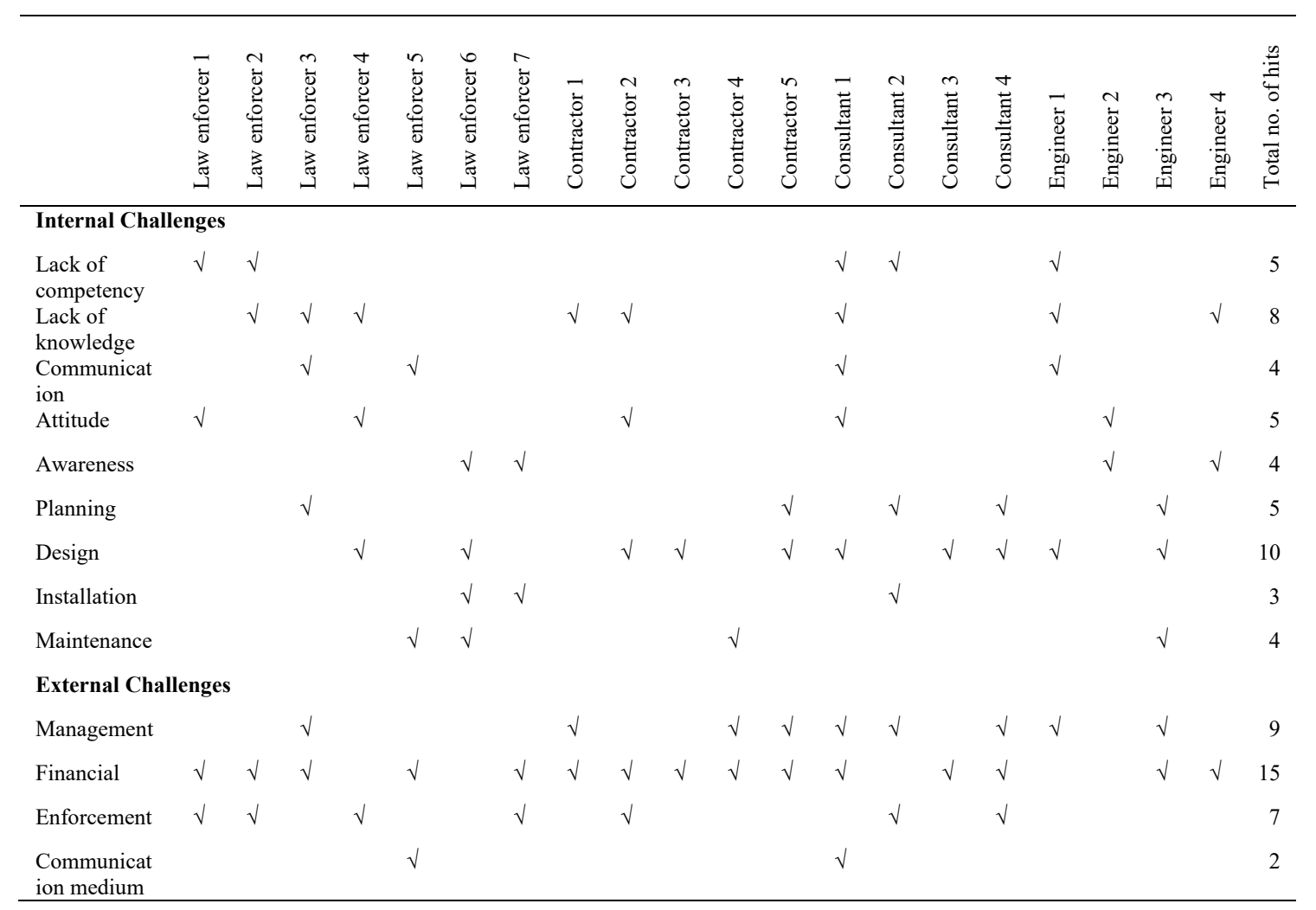

\subsubsection{External challenges}

Conversely, financial has the highest total number of hits among the identified external challenges in this study. Explicitly, several respondents stated that while the cost for implementing ESCP varies between different means and methods, there is a consensus that the cost for ESCP implementation is high. Additionally, some respondents report that there are projects with inadequate resources for implementing ESCP due to upper management constraints. Also, sometimes there is an insufficient 
budget allocated in contracts for maintaining specific ESCP devices. Therefore, industry practitioners will often choose plans with the lowest costs rather than using their experience to guide that decision. While some may argue that environmental officers may impact these decisions by providing the right advice, in reality, there are construction managers and project managers that avoid proper ESCP implementation to save money and reduce project cost. This situation can be avoided if project decisionmakers acquire adequate knowledge and awareness in environmental regulation for construction through formal training. However, providing training to individuals, especially the course for CPESC, is often cited as expensive. In conclusion, these findings suggest that construction companies are highly resistant to implementing environmental regulation because these organizations are profit-driven, and there is a lack of environmental awareness and culture for sustainable development.

\subsection{Success factors for implementing ESCP}

While the prior study suggests that four context-specific conditions impact the successful implementation of environmental regulation: policy operationalization, organizational position, professional belief, and specialist knowledge and understanding [18], this study's results show that the success factors for implementing one of the environmental laws in Malaysia can be categorized into four categories: strategic focus, people, operations, and finances. The subsequent subsections discuss each of those categories.

Table 3: Success factors in implementing ESCP

\begin{tabular}{|c|c|c|c|c|c|c|c|c|c|c|c|c|c|c|c|c|c|c|c|c|c|}
\hline & 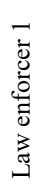 & 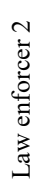 & 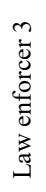 & 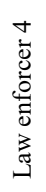 & 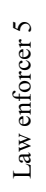 & 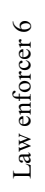 & 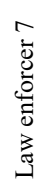 & 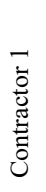 & 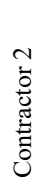 & 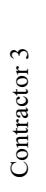 & 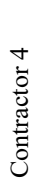 & 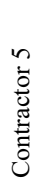 & 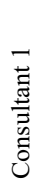 & 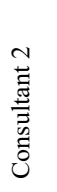 & 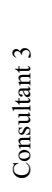 & 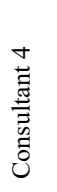 & 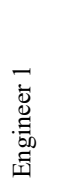 & 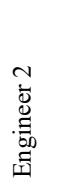 & 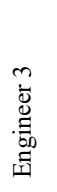 & 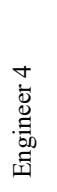 & 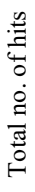 \\
\hline \multicolumn{22}{|c|}{ Strategic focus } \\
\hline Management & $\checkmark$ & $\checkmark$ & $\checkmark$ & $\checkmark$ & & $\checkmark$ & $\checkmark$ & & $\checkmark$ & $\checkmark$ & & $\sqrt{ }$ & $\checkmark$ & $\checkmark$ & $\checkmark$ & $\checkmark$ & v & $\checkmark$ & $\checkmark$ & $\checkmark$ & 17 \\
\hline Planning & & $\checkmark$ & $\checkmark$ & & & & & & & $\checkmark$ & & & & & & $\checkmark$ & & & & & 4 \\
\hline \multicolumn{22}{|l|}{ People } \\
\hline Knowledge & $\checkmark$ & $\checkmark$ & $\checkmark$ & $\checkmark$ & & $\checkmark$ & $\checkmark$ & & $\checkmark$ & $\checkmark$ & & & $\checkmark$ & & & & & & & & 7 \\
\hline $\begin{array}{l}\text { Communicati } \\
\text { on }\end{array}$ & & & & & & & & & & $\checkmark$ & & $\sqrt{ }$ & & & & & $\sqrt{ }$ & & & & 3 \\
\hline Attitude & $\checkmark$ & & & $\checkmark$ & $\checkmark$ & & & $\checkmark$ & $\checkmark$ & & & & $\checkmark$ & & & & & $\checkmark$ & $\checkmark$ & & 6 \\
\hline Awareness & & & & $\checkmark$ & & & & & & & & & $\checkmark$ & & $\checkmark$ & & & $\checkmark$ & $\checkmark$ & $\checkmark$ & 7 \\
\hline \multicolumn{22}{|l|}{ Operations } \\
\hline Designing & & & & $\checkmark$ & & $\checkmark$ & $\sqrt{ }$ & & $\checkmark$ & $\checkmark$ & $\sqrt{ }$ & & $\checkmark$ & & & $\checkmark$ & & $\checkmark$ & & & 6 \\
\hline Construction & & & & $\checkmark$ & & & & & & $\checkmark$ & & & v & & & & & & $\checkmark$ & & 5 \\
\hline Enforcement & $\checkmark$ & & $\checkmark$ & $\checkmark$ & $\checkmark$ & & & $\checkmark$ & $\checkmark$ & & $\checkmark$ & & $\checkmark$ & $\checkmark$ & $\checkmark$ & $\checkmark$ & & $\checkmark$ & $\checkmark$ & & 13 \\
\hline Maintaining & & & & & & & & & & & & & & & $\sqrt{ }$ & & & & $\checkmark$ & & 2 \\
\hline \multicolumn{22}{|l|}{ Finances } \\
\hline Budget & & & & & & $\checkmark$ & $\checkmark$ & & $\checkmark$ & $\checkmark$ & $\checkmark$ & $\sqrt{ }$ & & & & $\checkmark$ & & & & $\checkmark$ & 6 \\
\hline Design cost & & & & & & & & & & $\checkmark$ & $\checkmark$ & $\sqrt{ }$ & & & & & & & & $\checkmark$ & 5 \\
\hline $\begin{array}{l}\text { Equipment } \\
\text { cost }\end{array}$ & & & & & $\checkmark$ & & & $\checkmark$ & $\checkmark$ & & & & & & & $\checkmark$ & & & $\checkmark$ & & 4 \\
\hline Material cost & & & & & & & & & & & & $\sqrt{ }$ & & & & $\checkmark$ & & & & & 4 \\
\hline Labor cost & & & & & & $\checkmark$ & $\sqrt{ }$ & & & & & $\sqrt{ }$ & & & & & & & & & 3 \\
\hline
\end{tabular}




\subsubsection{Strategic focus}

Strategic focus consists of two factors - management and planning. Regarding management, developers need to appoint consultants and contractors that have adequate capabilities in implementing ESCP. Moreover, contractors need to hire individuals with knowledge of ESCP or train their employees on ESCP. Furthermore, in addition to providing mandatory training to environmental officers, upper management should also offer supplementary training to other organizations. Lastly, top management also plays a significant role in providing adequate resources to its environmental officers to deliver programs that improve awareness of ESCP among its employees. Conversely, for planning, appropriate project scheduling is essential to ensure that all project members understand their roles and responsibilities in implementing ESCP. In other words, these findings suggest that support from upper management and adequate planning will ease ESCP implementation by having project team members with the necessary knowledge and awareness on the importance of sustainability and environmental protection in construction.

\subsubsection{People}

People consist of factors such as knowledge, communication, attitude, and awareness. Specifically, participants suggest that research that ensures all parties know ESCP to avoid unnecessary misunderstandings is vital. For example, communication among construction parties is also critical because every decision will positively or negatively affect both the construction site and the off-site environment. Therefore, as a middleman between government authorities and contractors, consultants need to consult contractors actively on ESCP. Also, state representatives can play a more prominent role by becoming a medium for the community if any issue arises at construction sites. Effective communication on ESCP creates awareness regardless of whether the individuals are involved or not involved in the construction field. While participants suggest that construction projects hire skilled and experienced supervisors to construct ESCP devices, the implementation can only be successful only if every person related to the project has appropriate awareness and the right attitude. Primarily, participants suggest that developers, contractors, and consultants need to change their mindset and not take environmental regulation for granted. Therefore, full commitment from developers, consultants, and contractors' top management is necessary to ensure that everyone implements ESCP. Conversely, knowledge among individuals outside of the construction industry is also required. These individuals can play a role by reporting to responsible parties if any construction project does not adhere to environmental regulation. In other words, these findings suggest that everybody needs to know about environmental law to ensure the success of its implementation

\subsubsection{Operations}

Operations consist of design, construction, maintenance, and enforcement. Design, construction, and maintenance involve following the ESCP specification during the design phase and constructing and maintaining ESCP devices on-site as per that design during the project's execution phase. While those activities are necessary to have ESCP devices at construction sites, most participants suggest that enforcement plays a more significant factor in ensuring minimal misinformation and misunderstanding in ESCP implementation. Specifically, enforcement should provide awareness by inspecting all projects that cause erosion and sediment problems. Also, participants suggest that enforcement needs to be strict by having frequent monitoring of construction sites that perform earthwork activities. Specifically, while various guidelines are available to ensure minimal erosion and sedimentation at construction sites, several issues can be associated with enforcing the implementation itself by ensuring adherence to the specifications and design. Moreover, reducing misunderstanding among all parties is necessary to ensure that all the design and planning align with ESCP's objectives. 


\subsubsection{Finances}

Finances consist of budget, design cost, equipment cost, material cost, and labor cost that start from the contract, drawing, and design. Specifically, consultants need to provide the budget, cost, and plan for the project, and that allocation should be logical and reasonable. While government regulatory and incentive programs may drive positive outcomes, participants suggest that other initiatives that support client awareness on adopting sustainable construction practices should co-occur to ensure that program's effectiveness [19]. Therefore, without an appropriate awareness program for clients, consultants need to purposefully consider many matters when providing costing in terms of budget and design for installing and maintaining components of ESCP. In summary, allocating appropriate budget and costing during the planning phase is crucial as it ensures efficiency and positive outcome to support implementing ESCP in Malaysia.

\section{CONCLUSION}

To reduce the construction industry's impact on the environment, this study investigates the causal factors for successfully implementing environmental regulation by analyzing interview data with twenty industry practitioners involved in ESCP implementation in Malaysia. The major findings include:

- The challenges for ESCP implementation involve both internal and external challenges. Internal challenges involving project parties not performing their rightful responsibilities from the lack of knowledge and awareness of environmental protection. External challenges are caused by decisionmakers that take ESCP implementation for granted and do not see the value of implementing ESCP.

- The success factors for ESCP implementation relate to strategic focus, people, operations, and finances. Strategic focus involves managing and planning with integrity and responsibility. People consist of ensuring every person that is related to the project has knowledge and awareness of ESCP. The operation involves project parties following the specifications and design during constructing and maintaining ESCP devices. Finances include decision-makers allocating appropriate budget for the necessary means and methods.

These findings highlight the need to provide awareness and knowledge on the importance of environmental regulation. Specifically, policymakers can use the results to develop strategies for tackling problems related to ESCP implementation. Thus, this study's lesson would help the industry improve the success rate of environmental regulation. This research's fundamental theoretical contribution is by providing researchers and practitioners with a set of challenges and success factors for ESCP implementation.

\section{ACKNOWLEDGMENT}

This work is supported by Universiti Malaysia Pahang [RDU190382]. The authors would like to thank the editors and anonymous reviewers whose invaluable comments and suggestions substantially helped improve this paper's quality. The authors are also thankful to the industry practitioners that participated in this work.

\section{REFERENCES}

[1] Price, J. C., Karesh, R., "Tennessee erosion and sediment control handbook: a guide for protection of state waters through the use of best management practices during land disturbing activities," Tennessee Department of Environment and Conservation Division of Water Pollution Control. Tennessee Department of Environment and Conservation, 2002 
[2] Lowdermilk, J. M., Templeton, S. R., Privette III, C. V., Hayes, J. C., "An economic analysis of sediment control at construction sites: the case of Greenville County," South Carolina, No. 3212016-10755, 2011

[3] Wang, H. W., Kondolf, M., Tullos, D., Kuo, W. C., "Sediment management in Taiwan's reservoirs and barriers to implementation." Water, 10(8), 1034, 2018

[4] Mäki, T., "Multi-disciplinary discourse on design-related issues in construction site meetings." Procedia Economics and Finance, 21, 231-238, 2015

[5] Bamgbade, J. A., Kamaruddeen, A. M., Nawi, M. N. M., Adeleke, A. Q., Salimon, M. G., Ajibike, W. A., "Analysis of some factors driving ecological sustainability in construction firms. Journal of Cleaner Production," 208, 1537-1545, 2019

[6] Opoku, D. G. J., Ayarkwa, J., Agyekum, K., "Barriers to environmental sustainability of construction projects." Smart and Sustainable Built Environment, 2019

[7] Testa, F., Iraldo, F., Frey, M., "The effect of environmental regulation on firms' competitive performance: The case of the building \& construction sector in some EU regions." Journal of Environmental Management, 92(9), 2136-2144, 2011

[8] Fryrear, D. W., Skidmore, E. L., "Methods for controlling wind erosion. Soil erosion and crop productivity," 443-457, 1985

[9] Pimentel, D., "Soil erosion: a food and environmental threat." Environment, Development and Sustainability, 8(1), 119-137, 2006

[10] Fatt, C. S., "Sediment problems and their management in Peninsular Malaysia." Water International, 10(1), 3-6. 1985

[11] Devi, P., "Comparison of Consumption Patterns and Environmental Awareness in formal and informal communities in Suva, Fiji Islands," 2016

[12] Marttunen, M., Hämäläinen, R. P., "Decision analysis interviews in environmental impact assessment." European Journal of Operational Research, 87(3), 551-563, 1995

[13] Crouch, M., McKenzie, H., "The logic of small samples in interview-based qualitative research." Social Science Information, 45(4), 483-499, 2006

[14] Radzi, A.R., Bokhari, H.R., Rahman, R.A., Ayer, S.K., "Key attributes of change agents for successful technology adoptions in construction companies: a thematic analysis." In Computing in Civil Engineering 2019: Data, Sensing, and Analytics (pp. 430-437). Reston, VA: American Society of Civil Engineers, 2019

[15] Rahman, R. A., Ayer, S. K., "Prevalent issues in BIM-based construction projects." In Proceedings of Joint Conference on Computing in Construction (Vol. 1, pp. 645-652), 2019

[16] Chang, A. S., Shen, F. Y., Ibbs, W., "Design and construction coordination problems and planning for design-build project new users." Canadian Journal of Civil Engineering, 37(12), 1525-1534, 2010

[17] Ghisellini, P., Ripa, M., Ulgiati, S., "Exploring environmental and economic costs and benefits of a circular economy approach to the construction and demolition sector. A literature review." Journal of Cleaner Production, 178, 618-643, 2018

[18] Maund, K., Gajendran, T., Brewer, G., "Key issues for implementation of environmental planning policy: construction management practice.” Sustainability, 10(7), 2156, 2018

[19] Yin, B. C. L., Laing, R., Leon, M., Mabon, L., "An evaluation of sustainable construction perceptions and practices in Singapore." Sustainable Cities and Society, 39, 613-620, 2018 